\title{
Identification of Structurally Similar Phytochemicals to Quercetin with High SIRT1 Binding Affinity and Improving Diabetic Wound Healing by Using In silico
} Approaches

\author{
Rupal Dubey 1(i), Pranav Kumar Prabhakar ${ }^{2(\mathbb{D})}$, Jeena Gupta ${ }^{1, *(\mathbb{D})}$ \\ 1 Department of Biochemistry, School of Bioengineering and Biosciences, Lovely Professional University, Phagwara, \\ Punjab (India) - 144411 \\ 2 Department of Medical Laboratory Sciences, School of Physiotherapy and Paramedical Sciences, Lovely Professional \\ University, Phagwara, Punjab (India) - 144411 \\ * Correspondence: jeena.20104@1pu.co.in (J.G.);
}

Scopus Author ID 26648293400

Received: 15.09.2021; Revised: 10.11.2021; Accepted: 14.11.2021; Published: 26.11.2021

\begin{abstract}
Diabetes Mellitus is the most prevalent metabolic disorder that is increasing at an alarming rate worldwide. The unregulated glucose level leads to various types of health disorders, and one of the major diabetic complications is delayed wound healing. Due to the more side effects of synthetic drugs, there is a need to explore plants and their phytochemicals for medicinal purposes. It was found that Quercetin, a flavonoid, increases the rate of diabetic wound healing by enhancing the expression of SIRT1. This demands more insight towards Quercetin and its similar compounds, as it is hypothesized that similar compounds may have similar biological properties. Thus similarity searching was done to identify the most similar compounds of Quercetin, and then the molecular docking of the screened compounds was performed using AutoDock Vina. The unique ligands were docked into the active site of SIRT1 protein (PDB ID: 4ZZJ). The binding free energy of the interacting ligand with the protein was estimated. Six compounds were identified which possess the maximum structural similarity with Quercetin, and upon docking, it was found that gossypetin and herbacetin have similar binding modes and binding energy as that of Quercetin $(-7.5 \mathrm{kcal} / \mathrm{mol})$. Therefore, the hypothesis has been validated by in silico analysis. Our study identified two phytochemicals, Gossypetin, and Herbacetin which can prove beneficial for improving diabetic wound healing but needs to be validated further by in vitro and in vivo studies.
\end{abstract}

Keywords: diabetic wound healing; SIRT1; phytochemicals; similarity searching; molecular docking. (C) 2021 by the authors. This article is an open-access article distributed under the terms and conditions of the Creative Commons Attribution (CC BY) license (https://creativecommons.org/licenses/by/4.0/).

\section{Introduction}

Diabetes mellitus (DM) is a metabolic disorder characterized by raised blood sugar due to insufficient insulin secretion or action. Insulin is a hormone responsible for blood glucose regulation. Hyperglycemia is a prevalent impact of uncontrolled diabetes, resulting in serious damage to the body's nervous and blood vessel systems. Glucose is the body's primary energy source, and glucose management becomes irregular in the event of DM. The onset of DM has three significant flaws: increased glucose production in the liver, reduced pancreatic insulin secretion, and impaired insulin action. Symptoms include polyuria, polydipsia, constant hunger, weight loss, vision changes, and fatigue. DM is a critical disease that impacts 
contemporary society's quality of life and has recently increased globally [1]. Protein, fat, and carbohydrate metabolism complications are the most prevalent syndrome of this chronic disease and require adequate treatment [2].

Uncontrolled diabetes leads to many types of diseases, also referred to as diabetic complications, including diabetic retinopathy, neuropathy, nephropathy, foot ulcers, and delayed wound healing [3]. Many research studies have demonstrated that hyperglycemia is responsible for delayed wound healing, and it is one of the most serious complications of diabetes [4-7]. Hyperglycemia increases the oxidative stress that is accompanied by the enhanced production of free radicals [8,9]. The increased glucose level has been shown to decrease the rate of cell proliferation and collagen production, which increases the chances of tissue injury. Thus there is an urgent need to cure diabetes so that further complications of diabetes can be controlled.

For the treatment of diabetes and wound, several synthetic drugs are available in the market, but they are of high cost and have adverse side effects. The major limitation of these drugs is an allergic reaction and drug resistance [10]. The wound healing medicines have a major proportion of polypeptide growth factors which induces increased cell proliferation and eventually leads to cancer development [11]. The high cost, toxicity, and adverse effects of synthetic drugs highlight the need to identify new and effective treatment approaches for diabetic wounds [12]. Traditionally, plants and their phytochemicals were used throughout the world because of their effectiveness, relative abundance, low cost, and fewer side effects. Thus the use of such natural therapeutic drugs with the absence of any side effects appears promising towards chronic wound healing $[13,14]$.

Phytochemicals are non-nutritive chemicals produced by plants for self-protection and provide color and taste to fruits and vegetables [15]. Natural products contain a wide variety of chemicals, including flavonoids, polyphenols, saponins, steroids, and vitamins that have several therapeutic roles like anti-diabetic, antioxidant, anti-inflammatory, angiogenic, and cell synthesis modulating properties. The phytochemicals influence various metabolic pathways and thereby increase the release of insulin, it's production, and efficacy [16,17]. The literature survey reported that one of the most abundant plants flavonols is Quercetin which has various therapeutic effects.

Quercetin $\left(3,3^{\prime}, 4^{\prime}, 5,7\right.$-pentahydroxyflavone), is a naturally occurring poly-phenolic flavonoid [18]. It is a part of non-steroidal compounds derived from plants, known as phytoestrogens. A large amount of Quercetin occurs in fruits and vegetables like cranberry, onion, radish leaves, broccoli, nuts, seeds, etc. [19,20]. It may exist freely or be bound with sugars, but the conjugated form is better absorbed [21]. Thus, many common foods and beverages are rich sources of Quercetin and its derivatives [18]. Various clinical and nonclinical studies have shown strong anti-diabetic activity of Quercetin, and it is due to the more proliferation of pancreatic cells that enhance insulin secretion and glucose metabolism [22]. Quercetin enhances the expression of AMP-dependent protein kinase (AMPK) and Sirtuin 1 (SIRT1) that decreases glucose production [23]. SIRT1, also called Sirtuin (silent mating type information regulation 2 homolog) 1, is a class III histone deacetylase (HDAC) that removes an acetyl group from histone protein $\mathrm{N}$ terminal tails and thus regulates gene expression by regulating the accessibility of the transcription factors to the specific genes. In this way, SIRT1 regulates various cellular processes like inflammation, apoptosis, cellular senescence, oxidative stress, mitochondrial biogenesis, and function [24]. High glucose or high insulin resistance leads to reduced SIRT1 expression that can cause detrimental effects like 
increased oxidative stress, inflammation, impaired wound healing, etc. [25-27]. Thus potent SIRT1 activating compounds (SACs) need to be identified to overcome various diabetic complications.

Quercetin regulates various signal transduction pathways responsible for oxidative stress and thus acts as a scavenger of reactive oxygen species (ROS) [28]. ROS creates lethal effects and leads to various disorders like diabetes, cardiovascular diseases, and cancer [29]. Structurally presence of catechol groups in the ring $\mathrm{B}$ and free $\mathrm{OH}$ groups of the A ring and/or $\mathrm{C}$ ring is the result of its antioxidant activity [30]. The anti-inflammatory effect of Quercetin is due to the inhibition of enzymes responsible for triggering inflammation like lipoxygenase (LOX) and cyclooxygenase (COX) [31]. Thus Quercetin can improve wound healing by increasing fibroblast proliferation and reducing scar formation [32]. Quercetin also has other therapeutic effects as it acts as a potent anti-cancer agent [33]. It exerts its effect by altering the progression of the cell cycle, inhibiting the proliferation of cells, and promoting apoptosis [21,34].

In this study, we have evaluated various biological activities of Quercetin, and it has been found that Quercetin has no toxicity or side effects [35]. Thus due to the pronounced therapeutic activities of Quercetin, it has attracted more attention. Since nature is a vast reserve of phytochemicals that may have similar therapeutic activities as Quercetin but are still unexplored, there is a need to identify similar compounds that may cure diabetic wound healing. Therefore we hypothesize that structurally similar compounds can exhibit similar biological activities. Data mining and similarity searching was performed to identify the compounds similar to Quercetin. Further, Quercetin is known to enhance SIRT1 activity, so we also performed molecular docking of Quercetin and its similar compounds with SIRT1 (PDB ID: 4ZZJ) to predict their binding energies for a more confirmatory hypothesis.

\section{Materials and Methods}

\subsection{In silico studies: data mining and similarity searching.}

Quercetin was used as a query molecule for data mining and similarity searching in different open sources databases like CHEMBL, Protein Data Bank (PDB), PubChem, and DrugBank. 2D structure (Figure 1) and SMILE notation were used for searching the databases for more outputs. It was reported that if two molecules are structurally similar, then they may have the same biological activities [36,37].

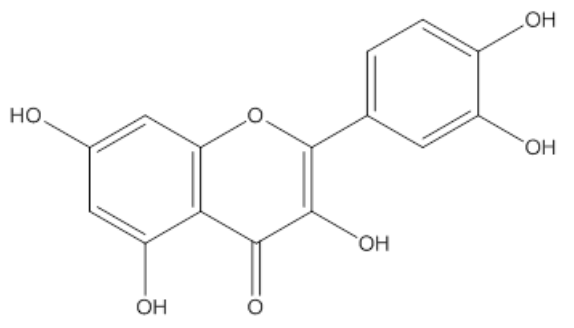

Figure 1. Structure of Quercetin.

\subsection{Molecular docking.}

Molecular docking is an in silico-approach for predicting the binding free energy of the query structure into the active site of the protein. AutoDockVina [38], a web-based server for 
docking, was used for the docking studies of Quercetin and its similar compounds into the binding site of PDB protein.

Protein Data Bank online database (http://www.rcsb.org/) was used to obtain the structure of human SIRT1 (PDB ID: 4ZZJ) in the pdb format [39]. Ligand and water molecules were removed while polar hydrogen and Kollman charge were added to SIRT1 (PDB ID: 4ZZJ) so as to convert it into pdbqt. Molecular docking of the bound ligand 4TQ i.e. (3S)-1,3dimethyl-N-[3-(1,3-oxazol-5-yl)phenyl]-6-[3-(trifluoromethyl)phenyl]-2,3-

dihydropyrido[2,3-b]pyrazine- $4(1 \mathrm{H})$-carboxamide) was performed to predict the binding energy of the reference molecule. Then the molecular docking of the molecules obtained by virtual screening was performed to evaluate the binding energy of each using AutoDock Vina. Certain parameters of docking was set, grid box 40 X 40 X 40 , grid centre $(-0.827,45.618$, 0.853 ) and grid spacing $0.375 \AA$. This study gives an idea about how similar molecules can bind to the active site of the same protein.

\subsection{PyMOL software.}

To better visualize the binding interactions of ligand and protein, the docking results are visualized using PyMOL software. This software also helps in predicting the distance between ligand and interacting amino acids.

\section{Results and Discussion}

Structural similarity searching identified various molecules with more than $80 \%$ similarity with Quercetin, out of which six compounds with the highest (>95\%) similarity (i.e., Myricetin, Quercetagetin, Gossypetin, 6-Hydroxykaempferol, Robinetin, and Herbacetin) were chosen for molecular docking (Table 1).

Table 1. Similarity searching using Quercetin as a query molecule.

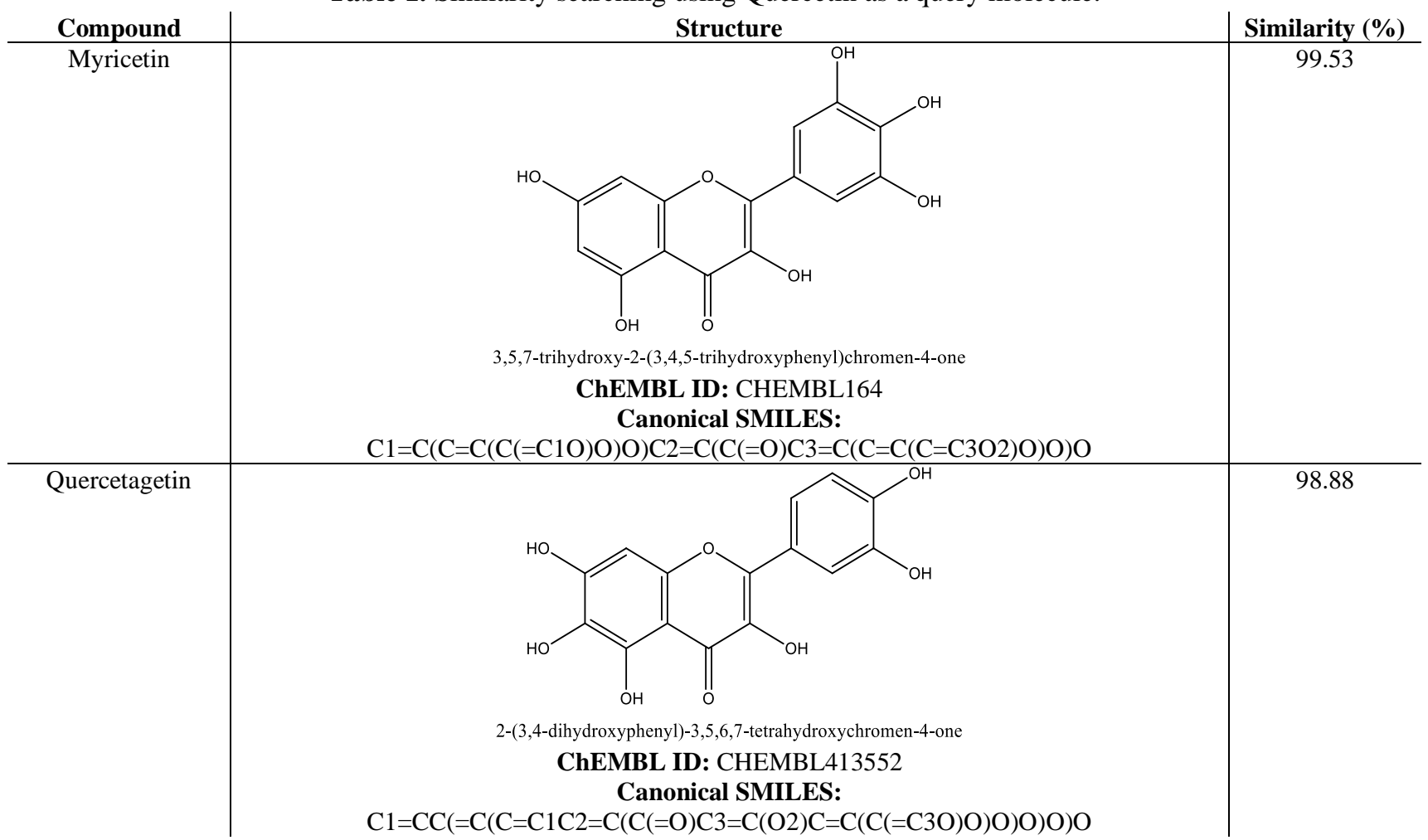




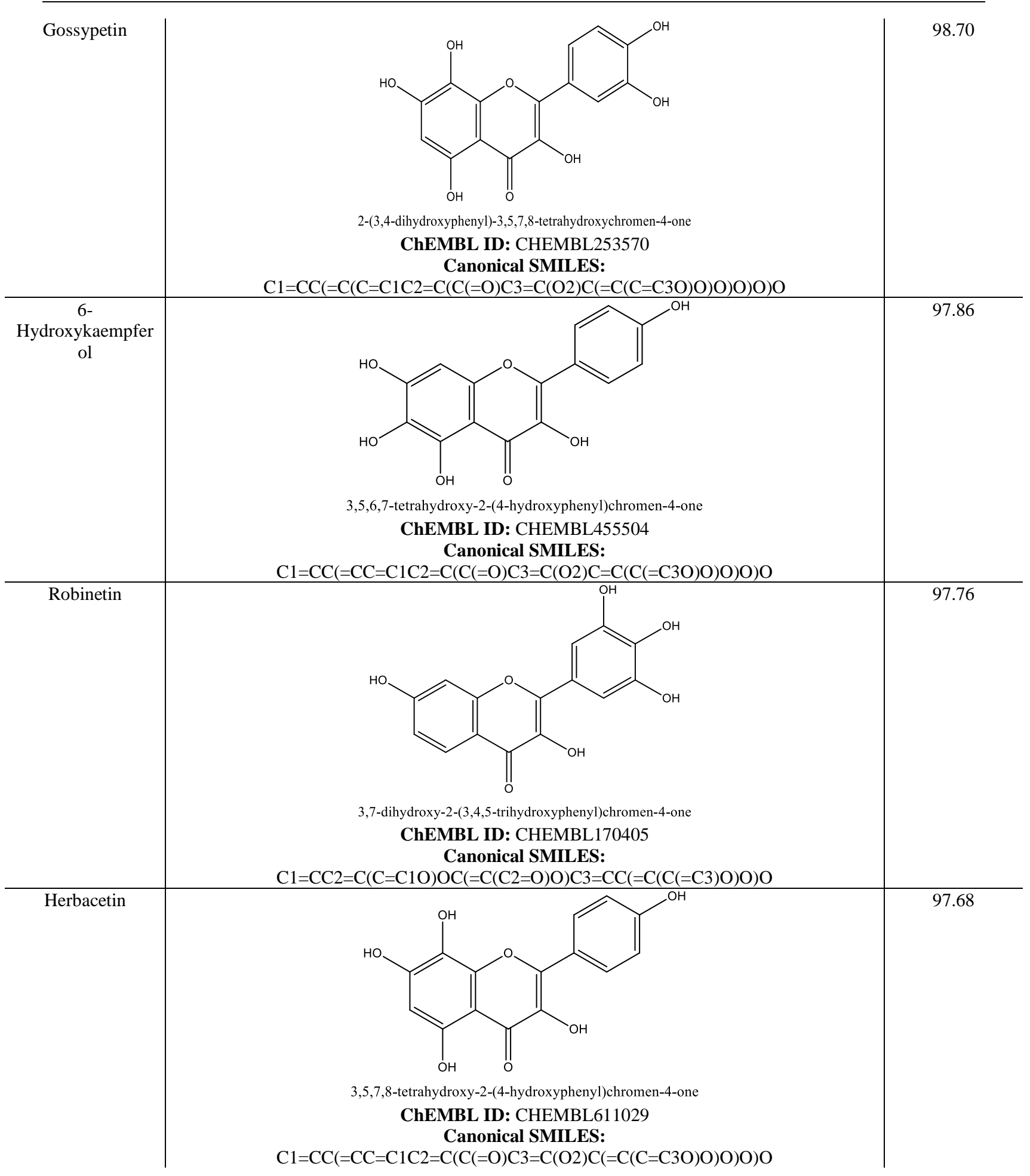

\subsection{Molecular docking.}

The redocking of the ligand 4TQ with the macromolecule 4ZZJ revealed an output of $8.0 \mathrm{kcal} / \mathrm{mol}$. Out of the six similar compounds, the binding energy of Gossypetin and Herbacetin is found to be $-7.5 \mathrm{kcal} / \mathrm{mol}$, which is similar to the binding affinity of the query structure, i.e., Quercetin (-7.5 kcal/mol) with SIRT1 (4ZZJ) (Table 2). Thus it can be hypothesized that Gossypetin and Herbacetin, having structural similarity with Quercetin and the same binding affinity towards 4ZZJ macromolecule (Figure 2), may have similar biological activities also. The docking results of bound ligand (4TQ), Gossypetin, and Herbacetin were visualized through PyMOL software for a better pictorial resolution of binding interactions (Figure 3, 4, 5). 
Table 2. Estimated binding free energy of the structurally similar compound with SIRT1 (PDB ID: 4ZZJ).

\begin{tabular}{|c|c|c|}
\hline Compound & Binding Free Energy (kcal/mol) & Interacting Amino Acids \\
\hline Quercetin (query structure) & -7.5 & $\begin{array}{l}\text { ARG274 } \\
\text { VAL657 } \\
\text { GLU467 } \\
\text { ARG649 } \\
\text { GLU477 } \\
\text { PHE474 } \\
\text { PRO470 } \\
\text { PRO468 } \\
\text { LEU469 }\end{array}$ \\
\hline Gossypetin & -7.5 & $\begin{array}{l}\text { VAL657 } \\
\text { ARG274 } \\
\text { LYS444 } \\
\text { PRO468 } \\
\text { LEU469 } \\
\text { SER442 }\end{array}$ \\
\hline Herbacetin & -7.5 & $\begin{array}{c}\text { LEU469 } \\
\text { PRO468 } \\
\text { HIS471 } \\
\text { LYS444 } \\
\text { ARG274 } \\
\text { VAL657 }\end{array}$ \\
\hline Myricetin & -7.2 & $\begin{array}{l}\text { ARG274 } \\
\text { VAL657 } \\
\text { LYS444 } \\
\text { SER442 } \\
\text { PRO468 } \\
\text { LEU469 }\end{array}$ \\
\hline 6-Hydroxykaempferol & -7.2 & $\begin{array}{c}\text { VAL657 } \\
\text { ARG274 } \\
\text { GLU467 } \\
\text { PRO468 } \\
\text { HIS471 } \\
\text { LEU469 }\end{array}$ \\
\hline Quercetagetin & -7.1 & $\begin{array}{c}\text { ARG199 } \\
\text { LEU202 } \\
\text { LYS203 } \\
\text { LEU206 } \\
\text { THR209 } \\
\text { ILE210 }\end{array}$ \\
\hline Robinetin & -7.1 & $\begin{array}{c}\text { GLU477 } \\
\text { ARG649 } \\
\text { PRO468 } \\
\text { PRO470 } \\
\text { HIS473 } \\
\text { PHE474 }\end{array}$ \\
\hline
\end{tabular}

Figure 2. Molecular docking of bound ligand 4TQ into the active site of protein (PDB ID:4ZZJ): (a) Binding interactions between the target protein and ligand (yellow); (b) Binding interactions between the surface view of ligand and secondary structure of target protein; (c) Visualization of binding interactions between target protein (cartoon structure) and ligand (magenta sphere) using Pymol software. 

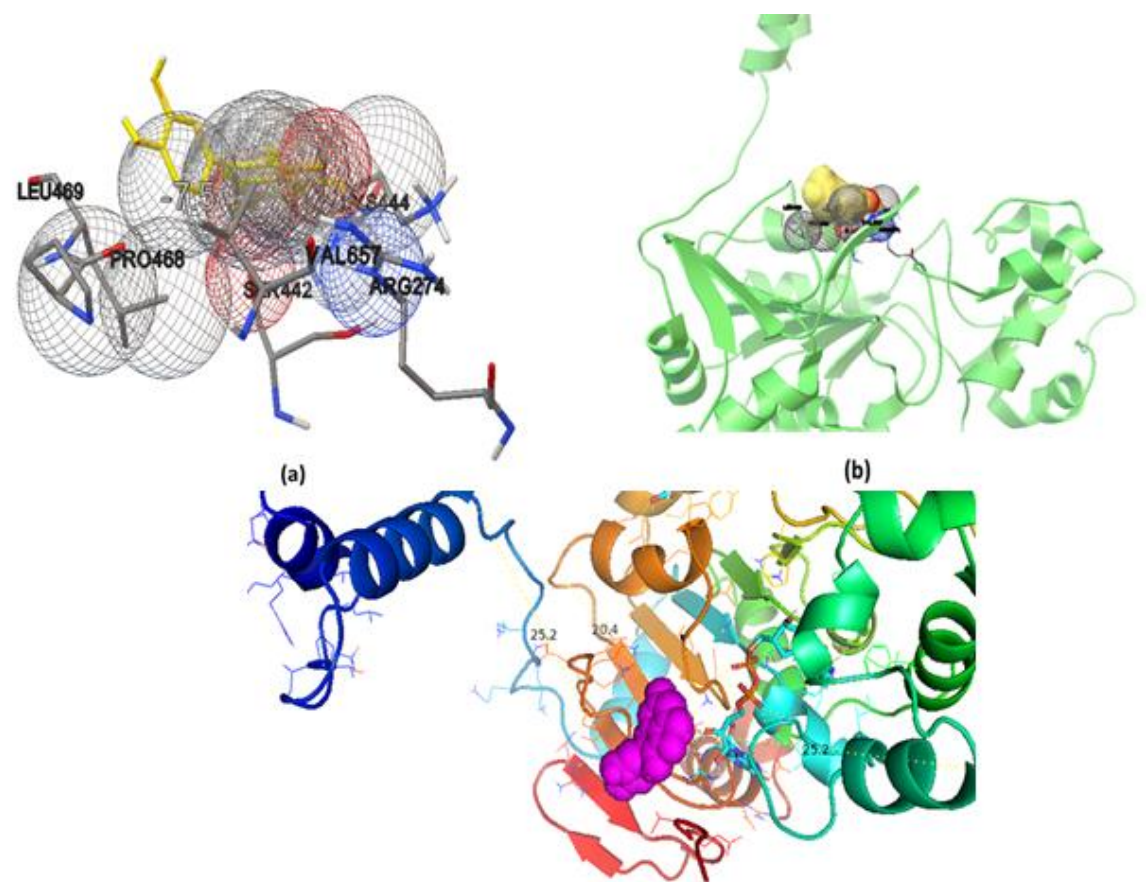

(c)

Figure 3. Molecular docking of query structure, i.e., Quercetin into the active site of protein (PDB ID:4ZZJ): (a) Binding interactions between the target protein and ligand (yellow); (b) Binding interactions between the surface view of ligand and secondary structure of target protein; (c) Visualization of binding interactions between target protein (cartoon structure) and ligand (magenta sphere) using Pymol software.

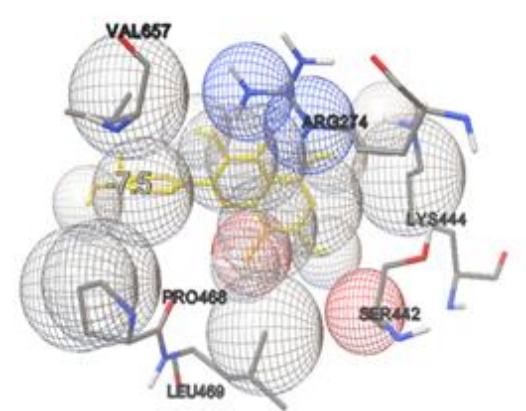

(a)

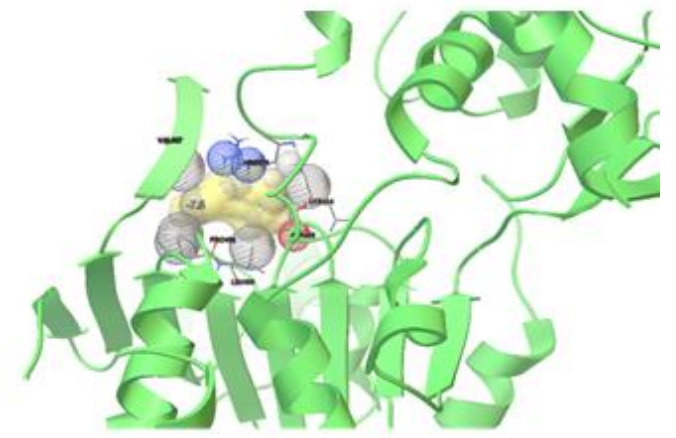

(b)

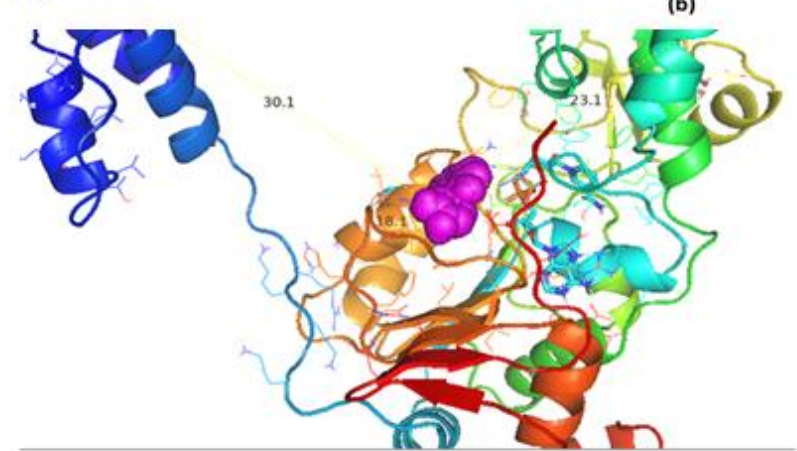

(c)

Figure 4. Molecular docking of unique ligand gossypetin into the active site of protein (PDB ID:4ZZJ): (a) Binding interactions between the target protein and ligand (yellow); (b) Binding interactions between the surface view of ligand and secondary structure of target protein; (c) Visualization of binding interactions between target protein (cartoon structure) and ligand (magenta sphere) using Pymol software. 


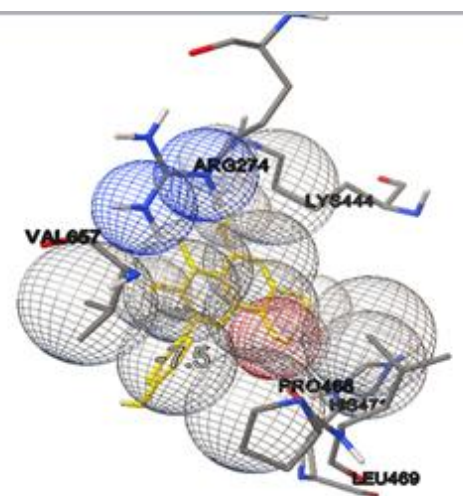

(a)

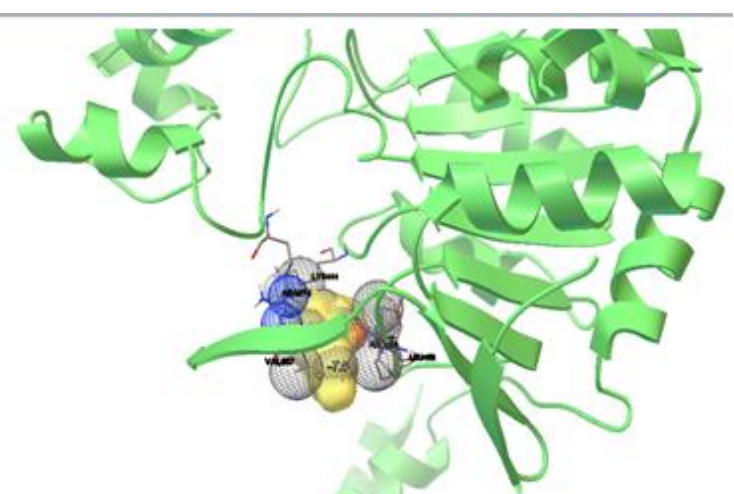

(b)

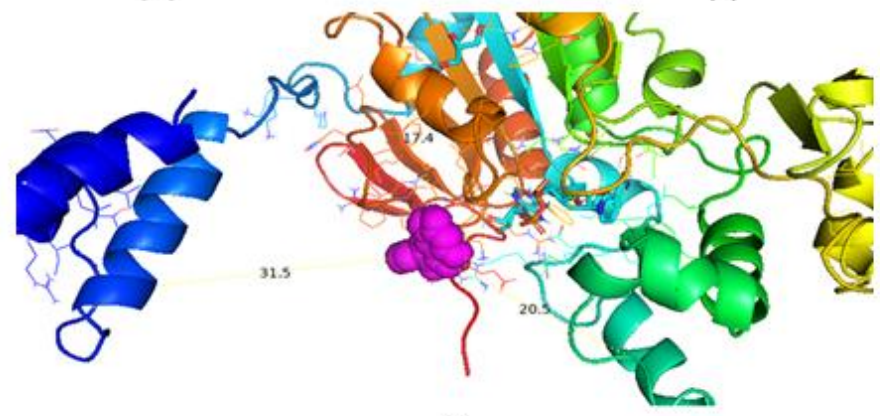

(c)

Figure 5. Molecular docking of unique ligand Herbacetin into the active site of protein (PDB ID:4ZZJ): (a) Binding interactions between the target protein and ligand (yellow); (b) Binding interactions between the surface view of ligand and secondary structure of target protein; (c) Visualization of binding interactions between target protein (cartoon structure) and ligand (magenta sphere) using Pymol software.

\subsection{Discussion.}

The occurrence of chronic wounds is a significant problem for diabetic people, which increases healthcare costs [40]. No effective strategies are available for the treatment of this complication. Currently, SIRT1 is emerging as an important therapeutic target for increasing diabetic wound healing. Lower expression of SIRT1 was observed in streptozocin (STZ)induced diabetic mice and diabetes patients with reduced wound healing. Treating the wounds with a synthetic SIRT1 activator (SRT1720) locally increased angiogenesis and improved wound healing. Furthermore, SRT1720 was also found to increase the proliferation, migration, and in vitro tube formation ability by human umbilical vein endothelial cells (HUVECs). The SIRT1 activator mediates the protective effect by reducing oxidative stress injury [41].

Many natural phytochemicals are evaluated as effective SACs. Reports have emphasized that dietary supplementation of polyphenols enhances the deacetylase activity of SIRT1 and protects against metabolic diseases. Traditional Chinese medicine includes many active molecules which are potent SIRT1 activators [42]. Diabetes-associated impaired tissue regeneration, and reduced wound healing often gets translated to end-stage complications like diabetic foot disease and foot ulceration. It was estimated by the International Diabetes Federation (IDF, 2015) that around 9.1 million to 26.1 million diabetic people develop foot ulcers every year worldwide [43]. Endothelial progenitor cells (EPCs) are a central role player in angiogenesis, and their dysfunction is directly linked with diabetic wound healing [44]. They significantly contribute to tissue repair by migrating to different sites and proliferating to form new capillaries from the previous ones on receiving angiogenic stimuli [45]. Quercetin, a natural flavonoid, had been reported to protect EPCs against high glucose-induced impaired cell migration by increasing SIRT1 expression and Sirt1-dependent eNOS upregulation. 
Sirtinol, a chemical antagonist of SIRT1, abolished these protective effects of Quercetin [46]. Due to the beneficial roles of Quercetin, similarity searching was done to identify similar compounds. Since the similarity index is chosen to be more than $80 \%$, which means the compounds whose structure resembles $80 \%$ and more to Quercetin will be fetched, six compounds with maximum similarity with Quercetin were chosen. The similar compounds were then docked with SIRT1 protein (PDB ID: 4ZZJ) to evaluate the binding energies. The study showed that similar compounds interacted in the same manner as the query structure into the active site of 4ZZJ. The interacting amino acids present in the active site of bound ligand, i.e., 4TQ and Quercetin, are the same, which infers Quercetin is also binding with the SIRT1 activator protein just like 4TQ. Also, the resemblance of amino acids with Quercetin is found in the identified phytochemicals (PRO468, LEU469, VAL657, ARG274); thus, it is predicted that those phytochemicals will have the same properties as that of Quercetin since their binding interactions are nearly the same. Some of the interacting amino acids are hydrophobic (VAL657, LEU469, PRO468, PHE474), while some are hydrophilic (ARG274, THR209, GLU467) in nature, and they show van der Waals interaction and few hydrogen bonds while binding with the ligand. Quercetin or similar phytochemicals are SIRT1 activators that show HDAC activity. Thus they promote transcription of various signaling molecules that help in improving diabetic wound healing.

The above study revealed that similar structures (Gossypetin and Herbacetin) are binding with the SIRT1 activator protein equivalent to Quercetin; thus, the in silico analysis gives an insight that the similar compounds will have the same biological activities. Gossypetin is a hexahydroxyl flavone, first isolated from the Hibiscus species ( $H$. vitifolius and $H$. furcatus). The plant extracts were traditionally used to cure diabetes, jaundice, and inflammation and possess antioxidant activity [47-49]. Herbacetin, a pentahydroxyl flavone, occurs naturally in Rhodiola rosea [50] and cotton [51]. Herbacetin shows antioxidant, antitumor [52,53] and anti-inflammatory properties [54]. The diabetic wound healing activity of Herbacetin and Gossypetin as of Quercetin have been proved through in silico analysis and can be further investigated through in vivo and in vitro analysis.

\section{Conclusions}

Nutraceuticals and phytochemicals are emerging as promising elements for promoting health due to fewer side effects, relative abundance, and less cost. Several plant secondary metabolites like stilbenes, flavones, anthocyanidins, and chalcones are best known for their beneficial therapeutic activities. Our study highlights the biological activities of phytochemicals Herbacetin and Gossypetin, which possess high structural similarity with biologically active Quercetin and thus may prove beneficial for improving diabetic wound healing. Both the phytochemicals also possess a high binding affinity with the active site of SIRT1, similar to Quercetin. To the best of our knowledge, this is the first report to identify Quercetin's similar compounds and evaluate their binding energies using the molecular docking approach and further validating the results through PyMOL visualization. Further in vitro and in vivo analysis of these similar identified compounds can authenticate the in silico results.

\section{Funding}

This research received no external funding. 


\section{Acknowledgments}

The authors sincerely thank Lovely Professional University, Punjab, India, for providing the necessary facilities to carry out the study.

\section{Conflicts of Interest}

The authors declare no conflict of interest.

\section{References}

1. McGuire, S. Centers for Disease Control and Prevention. State Indicator Report on Physical Activity, 2014. Atlanta, GA: U.S. Department of Health and Human Services; 2014. Advances in Nutrition 2014, 5, 762-763, https://doi.org/10.3945/an.114.007211.

2. Bremer, A.A.; Mietus-Snyder, M.; Lustig, R.H. Toward a unifying hypothesis of metabolic syndrome. Pediatrics 2012, 129, 557-570, https://doi.org/10.1542/peds.2011-2912.

3. Berlanga-Acosta, J.; Mendoza-Mari, Y.; Martínez, M.D.; Valdés-Perez, C.; Ojalvo, A.G.; Armstrong, D.G. Expression of cell proliferation cycle negative regulators in fibroblasts of an ischemic diabetic foot ulcer. A clinical case report. International wound journal 2013, 10, 232-236, https://doi.org/10.1111/j.1742481X.2012.12000.x.

4. Ishihara, J.; Ishihara, A.; Fukunaga, K.; Sasaki, K.; White, M.J.; Briquez, P.S.; Hubbell, J.A. Laminin heparinbinding peptides bind to several growth factors and enhance diabetic wound healing. Nature communications 2018, 9, 1-14, https://doi.org/10.1038/s41467-018-04525-w.

5. Zhang, X.-N.; Ma, Z.-J.; Wang, Y.; Sun, B.; Guo, X.; Pan, C.-Q.; Chen, L.-M. Angelica Dahurica ethanolic extract improves impaired wound healing by activating angiogenesis in diabetes. PloS one 2017, 12, https://doi.org/10.1371/journal.pone.0177862.

6. Kido, D.; Mizutani, K.; Takeda, K.; Mikami, R.; Matsuura, T.; Iwasaki, K.; Izumi, Y. Impact of diabetes on gingival wound healing via oxidative stress. PLoS One 2017, 12, https://doi.org/10.1371/journal.pone.0189601.

7. Hu, S.C.-S.; Lan, C.-C.E. High-glucose environment disturbs the physiologic functions of keratinocytes: focusing on diabetic wound healing. Journal of Dermatological Science 2016, 84, 121-127, https://doi.org/10.1016/j.jdermsci.2016.07.008.

8. Brownlee, M. The pathobiology of diabetic complications: a unifying mechanism. diabetes 2005, 54, 16151625, https://doi.org/10.2337/diabetes.54.6.1615.

9. Kwon, Y.-I.I.; Vattem, D.A.; Shetty, K. Evaluation of clonal herbs of Lamiaceae species for management of diabetes and hypertension. Asia pacific journal of clinical nutrition 2006, 15.

10. Kim, J.; Lee, C.-M. Wound healing potential of a polyvinyl alcohol-blended pectin hydrogel containing Hippophae rahmnoides L. extract in a rat model. International journal of biological macromolecules 2017, 99, 586-593, https://doi.org/10.1016/j.ijbiomac.2017.03.014.

11. Min, D.; Nube, V.; Tao, A.; Yuan, X.; Williams, P.F.; Brooks, B.A.; Wong, J.; Twigg, S.M.; McLennan, S.V. Monocyte phenotype as a predictive marker for wound healing in diabetes-related foot ulcers. Journal of Diabetes and its Complications 2021, 35, https://doi.org/10.1016/j.jdiacomp.2021.107889.

12. Greenwald, M.B.-Y.; Tacconi, C.; Jukic, M.; Joshi, N.; Hiebert, P.; Brinckmann, J.; Tenor, H.; Naef, R.; Werner, S. A Dual-Acting Nitric Oxide Donor and Phosphodiesterase 5 Inhibitor Promotes Wound Healing in Normal Mice and Mice with Diabetes. Journal of Investigative Dermatology 2021, 141, 415-426, https://doi.org/10.1016/j.jid.2020.05.111.

13. Ribeiro, M.C.; Correa, V.L.R.; da Silva, F.K.L.; Casas, A.A.; das Chagas, A.d.L.; de Oliveira, L.P.; Miguel, M.P.; Diniz, D.G.A.; Amaral, A.C.; de Menezes, L.B. Wound healing treatment using insulin within polymeric nanoparticles in the diabetes animal model. European Journal of Pharmaceutical Sciences 2020, 150, https://doi.org/10.1016/j.ejps.2020.105330.

14. Nabavi, S.F.; Braidy, N.; Gortzi, O.; Sobarzo-Sanchez, E.; Daglia, M.; Skalicka-Woźniak, K.; Nabavi, S.M. Luteolin as an anti-inflammatory and neuroprotective agent: A brief review. Brain research bulletin 2015, 119, 1-11. https://doi.org/10.1016/j.brainresbull.2015.09.002.

15. Sayahi, M.; Shirali, S. The anti-diabetic and antioxidant effects of carotenoids: a review. Asian Journal of Pharmaceutical Research and Health Care 2017, 9, 186-191, https://doi.org/10.18311/ajprhc/2017/7689.

16. Prabhakar, P.K.; Doble, M. Synergistic effect of phytochemicals in combination with hypoglycemic drugs on glucose uptake in myotubes. Phytomedicine 2009, 16, 1119-1126, https://doi.org/10.1016/j.phymed.2009.05.021.

17. Salla, H.R.; Al Habsi, F.S.; Al Sharji, W.H. A comparative study on the role of Omani honey with various food supplements on diabetes and wound healing. Journal of King Saud University-Science 2020, 32, 2122 2128, https://doi.org/10.1016/j.jksus.2020.02.016. 
18. D'Andrea, G. Quercetin: a flavonol with multifaceted therapeutic applications? Fitoterapia 2015, 106, 256271, https://doi.org/10.1016/j.fitote.2015.09.018.

19. Bischoff, S.C. Quercetin: potentials in the prevention and therapy of disease. Current Opinion in Clinical Nutrition \& Metabolic Care 2008, 11, 733-740, https://doi.org/10.1097/MCO.0b013e32831394b8.

20. Patel, R.V.; Mistry, B.M.; Shinde, S.K.; Syed, R.; Singh, V.; Shin, H.-S. Therapeutic potential of Quercetin as a cardiovascular agent. European journal of medicinal chemistry 2018, 155, 889-904, https://doi.org/10.1016/j.ejmech.2018.06.053.

21. Tang, S.-M.; Deng, X.-T.; Zhou, J.; Li, Q.-P.; Ge, X.-X.; Miao, L. Pharmacological basis and new insights of quercetin action in respect to its anti-cancer effects. Biomedicine \& Pharmacotherapy 2020, 121, https://doi.org/10.1016/j.biopha.2019.109604.

22. Mahabady, M.K.; Shamsi, M.M.; Ranjbar, R.; Tabandeh, M.R.; Khazaeel, K. Quercetin improved histological structure and upregulated adiponectin and adiponectin receptors in the placenta of rats with gestational diabetes mellitus. Placenta 2021, 106, 49-57, https://doi.org/10.1016/j.placenta.2021.02.008.

23. Khursheed, R.; Singh, S.K.; Wadhwa, S.; Gulati, M.; Awasthi, A. Enhancing the potential preclinical and clinical benefits of Quercetin through novel drug delivery systems. Drug discovery today 2019, 25, 209-222, https://doi.org/10.1016/j.drudis.2019.11.001.

24. Prabhakar, P.K.; Singh, K.; Kabra, D.; Gupta, J. Natural SIRT1 modifiers as promising therapeutic agents for improving diabetic wound healing. Phytomedicine 2020, 76, https://doi.org/10.1016/j.phymed.2020.153252.

25. Sun, C.; Zhang, F.; Ge, X.; Yan, T.; Chen, X.; Shi, X.; Zhai, Q. SIRT1 improves insulin sensitivity under insulin-resistant conditions by repressing PTP1B. Cell metabolism 2007, 6, 307-319, https://doi.org/10.1016/j.cmet.2007.08.014.

26. Bo, S.; Togliatto, G.; Gambino, R.; Ponzo, V.; Lombardo, G.; Rosato, R.; Cassader, M.; Brizzi, M.F. Impact of sirtuin-1 expression on H3K56 acetylation and oxidative stress: a double-blind randomized controlled trial with resveratrol supplementation. Acta diabetologica 2018, 55, 331-340, https://doi.org/10.1007/s00592-0171097-4.

27. Peng, J.; Zhou, Y.; Deng, Z.; Zhang, H.; Wu, Y.; Song, T.; Yang, Y.; Wei, H.; Peng, J. miR-221 negatively regulates inflammation and insulin sensitivity in white adipose tissue by repression of sirtuin-1 (SIRT1). Journal of cellular biochemistry 2018, 119, 6418-6428, https://doi.org/10.1002/jcb.26589.

28. Xu, D.; Hu, M.-J.; Wang, Y.-Q.; Cui, Y.-L. Antioxidant activities of Quercetin and its complexes for medicinal application. Molecules 2019, 24, https://doi.org/10.3390/molecules24061123.

29. Valko, M.; Rhodes, C.; Moncol, J.; Izakovic, M.; Mazur, M. Free radicals, metals and antioxidants in oxidative stress-induced cancer. Chemico-biological interactions 2006, 160, 1-40, https://doi.org/10.1016/j.cbi.2005.12.009.

30. Jaishree, V.; Narsimha, S. Swertiamarin and quercetin combination ameliorates hyperglycemia, hyperlipidemia and oxidative stress in streptozotocin-induced type 2 diabetes mellitus in wistar rats. Biomedicine \& Pharmacotherapy 2020, 130, https://doi.org/10.1016/j.biopha.2020.110561.

31. Lesjak, M.; Beara, I.; Simin, N.; Pintać, D.; Majkić, T.; Bekvalac, K.; Orčić, D.; Mimica-Dukić, N. Antioxidant and anti-inflammatory activities of Quercetin and its derivatives. Journal of Functional Foods 2018, 40, 68-75, https://doi.org/10.1016/j.jff.2017.10.047.

32. Jangde, R.; Srivastava, S.; Singh, M.R.; Singh, D. In vitro and In vivo characterization of Quercetin loaded multiphase hydrogel for wound healing application. International journal of biological macromolecules 2018, 115, 1211-1217, https://doi.org/10.1016/j.ijbiomac.2018.05.010.

33. Choudhary, A.; Kant, V.; Jangir, B.L.; Joshi, V. Quercetin loaded chitosan tripolyphosphate nanoparticles accelerated cutaneous wound healing in Wistar rats. European journal of pharmacology 2020, 880, https://doi.org/10.1016/j.ejphar.2020.173172.

34. Massi, A.; Bortolini, O.; Ragno, D.; Bernardi, T.; Sacchetti, G.; Tacchini, M.; De Risi, C. Research progress in the modification of quercetin leading to anticancer agents. Molecules 2017, 22, https://doi.org/10.3390/molecules22081270.

35. Ebrahimpour, S.; Zakeri, M.; Esmaeili, A. Crosstalk between obesity, diabetes, and alzheimer's disease: Introducing Quercetin as an effective triple herbal medicine. Ageing Research Reviews 2020, 62, https://doi.org/10.1016/j.arr.2020.101095.

36. Martin, Y.C.; Kofron, J.L.; Traphagen, L.M. Do structurally similar molecules have similar biological activity? Journal of medicinal chemistry 2002, 45, 4350-4358, https://doi.org/10.1021/jm020155c.

37. Güller, P. The In Vitro and In Silico Inhibition Mechanism of Glutathione Reductase by Resorcinol Derivatives: A Molecular Docking Study. Journal of Molecular Structure 2021, 1228, https://doi.org/10.1016/j.molstruc.2020.129790.

38. Grosdidier, A.; Zoete, V.; Michielin, O. SwissDock, a protein-small molecule docking web service based on EADock DSS. Nucleic acids research 2011, 39, W270-W277, https://doi.org/10.1093/nar/gkr366.

39. Dai, H.; Case, A.W.; Riera, T.V.; Considine, T.; Lee, J.E.; Hamuro, Y.; Zhao, H.; Jiang, Y.; Sweitzer, S.M.; Pietrak, B. Crystallographic structure of a small molecule SIRT1 activator-enzyme complex. Nature communications 2015, 6, 1-10, https://doi.org/10.1038/ncomms8645. 
40. Song, R.; Mao, X.; Tang, S. $\kappa / \beta$-Carrageenan oligosaccharides promoting polarization of LPS-activated macrophage and their potential in diabetes wound healing. Materials Science and Engineering: C 2021, 121, https://doi.org/10.1016/j.msec.2020.111830.

41. Li, W.; Kandhare, A.D.; Mukherjee, A.A.; Bodhankar, S.L. Hesperidin, a plant flavonoid accelerated the cutaneous wound healing in streptozotocin-induced diabetic rats: Role of TGF-ß/Smads and Ang-1/Tie-2 signaling pathways. EXCLI journal 2018, 17, https://doi.org/10.17179/excli2018-1036.

42. Feng, B.; Huang, B.; Jing, Y.; Shen, S.; Feng, W.; Wang, W.; Meng, R.; Zhu, D. Silymarin ameliorates the disordered glucose metabolism of mice with diet-induced obesity by activating the hepatic SIRT1 pathway. Cellular Signalling 2021, 84, https://doi.org/10.1016/j.cellsig.2021.110023.

43. Armstrong, D.G.; Boulton, A.J.; Bus, S.A. Diabetic foot ulcers and their recurrence. New England Journal of Medicine 2017, 376, 2367-2375, https://doi.org/10.1056/NEJMra1615439.

44. El Ashram, S.; El-Samad, L.M.; Basha, A.A.; El Wakil, A. Naturally-derived targeted therapy for wound healing: Beyond classical strategies. Pharmacological Research 2021, 170, https://doi.org/10.1016/j.phrs.2021.105749.

45. Potente, M.; Gerhardt, H.; Carmeliet, P. Basic and therapeutic aspects of angiogenesis. Cell 2011, 146, 873887, https://doi.org/10.1016/j.cell.2011.08.039.

46. Zhao, L.-R.; Du, Y.-J.; Chen, L.; Liu, Z.-G.; Pan, Y.-H.; Liu, J.-F.; Liu, B. Quercetin protects against high glucose-induced damage in bone marrow-derived endothelial progenitor cells. International journal of molecular medicine 2014, 34, 1025-1031, https://doi.org/10.3892/ijmm.2014.1852.

47. Patel, K.; Patel, D.K. Therapeutic Benefit and Pharmacological Activities of Gossypetin: Biological Importance in the Medicine through Scientific Research Data Analysis. Metabolism-Clinical and Experimental 2021, 116, https://doi.org/10.1016/j.metabol.2020.154550.

48. Vijayan, P.; Raghu, C.; Ashok, G.; Dhanaraj, S.; Suresh, B. Antiviral activity of medicinal plants of Nilgiris. Indian Journal of medical research 2004, 120, 24-29.

49. Cai, Z.; Li, X.; Katsumura, Y. Interaction of hydrated electron with dietary flavonoids and phenolic acids:: Rate constants and transient spectra studied by pulse radiolysis. Free radical biology and medicine 1999, 27, 822-829, https://doi.org/10.1016/S0891-5849(99)00118-5.

50. Pangarova, T.; Zapesochnaya, G.; Nukhimovskii, E. Flavonoids of Rhodiola algida. Chemistry of Natural Compounds 1974, 10, 685-686.

51. Neelakantam, K.; Seshadri, T. Pigments of cotton flowers. PartIV. Constitution of herbacitrin and herbacetinnew glucoside and aglucone (flavonol). In: Proceedings of the Proc Indian Acad Sci-Section A. 1937; pp. 357-364.

52. Kim, D.J.; Lee, M.-H.; Liu, K.; Lim, D.Y.; Roh, E.; Chen, H.; Kim, S.-H.; Shim, J.-H.; Kim, M.O.; Li, W. Herbacetin suppresses cutaneous squamous cell carcinoma and melanoma cell growth by targeting AKT and ODC. Carcinogenesis 2017, 38, 1136-1146, https://doi.org/10.1093/carcin/bgx082.

53. Kim, D.J.; Roh, E.; Lee, M.-H.; Oi, N.; Kim, M.O.; Cho, Y.-Y.; Pugliese, A.; Shim, J.-H.; Chen, H.; Cho, E.J. Herbacetin is a novel allosteric inhibitor of ornithine decarboxylase with antitumor activity. Cancer research 2016, 76, 1146-1157, https://doi.org/10.1158/0008-5472.can-15-0442.

54. Li, L.; Sapkota, M.; Kim, S.-W.; Soh, Y. Herbacetin inhibits RANKL-mediated osteoclastogenesis in vitro and prevents inflammatory bone loss in vivo. European Journal of Pharmacology 2016, 777, 17-25, https://doi.org/10.1016/j.ejphar.2016.02.057. 